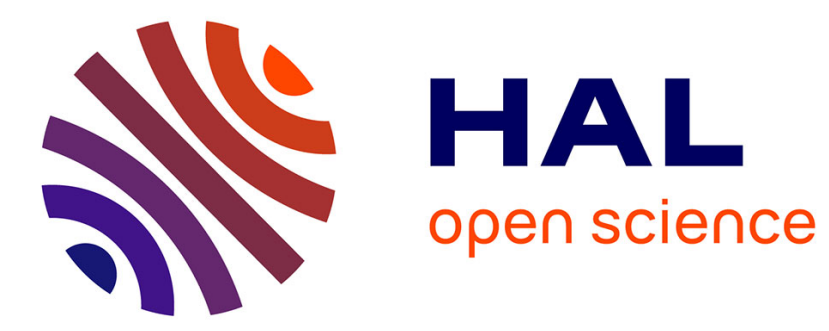

\title{
Sur la lumière positive et le passage de l'électricité dans les gaz \\ P. Villard
}

\section{To cite this version:}

P. Villard. Sur la lumière positive et le passage de l'électricité dans les gaz. J. Phys. Theor. Appl., 1908, 7 (1), pp.325-361. 10.1051/jphystap:019080070032500 . jpa-00241308

\section{HAL Id: jpa-00241308 https://hal.science/jpa-00241308}

Submitted on 1 Jan 1908

HAL is a multi-disciplinary open access archive for the deposit and dissemination of scientific research documents, whether they are published or not. The documents may come from teaching and research institutions in France or abroad, or from public or private research centers.
L'archive ouverte pluridisciplinaire HAL, est destinée au dépôt et à la diffusion de documents scientifiques de niveau recherche, publiés ou non, émanant des établissements d'enseignement et de recherche français ou étrangers, des laboratoires publics ou privés. 
VILLARD. - PASSAGE DE L'ÉLEGTRIGITÉ DANS LES GAZ 325

SUR LA LUMIÈRE POSITIVE ET LE PASSAGE DE L'ÉLECTRICITÉ DANS LES GAZ;

Par M. P. VILLARD.

On admet volontiers que le passage de l'électricité dans un gaz se fait exclusivement par le double phénomène de l'ionisation de ce gaz et de la recombinaison des ions mis en liberté. Dans cette dissociation moléculaire, les corpuscules cathodiques jouent un rôle essentiel : les vitesses considérables qu'ils acquièrent dès qu'un champ est établi dans le gaz leur permettent d'ioniser un grand nombre de molécules, d'en détacher de nouveaux corpuscules qui, sans avoir besoin de partir de la cathode, peuvent jouer le même rôle que les premiers; les ions positifs, constitués par toutes les molécules privées d'un corpuscule négatif, prennent également, sous l'action de la force électrique, une vitesse qui leur permet, si cette force est suffisante, d'ioniser également de nouvelles molécules. Une rapide multiplication des charges libres se produit ainsi, et le courant de convection qu'elles déterminent acquiert en un temps très court une intensité considérable. D'après cette manière de voir, la lumière émise par le gaz résulte nécessairement soit de la dissociation des molécules, soit de la recombinaison des ions libérés.

Cette conclusion paraît cependant être en désaccord avec un certain nombre de faits: Ainsi le voisinage d'un corps radioactif, cause certaine d'ionisation, facilite bien le passage de l'étincelle entre les boules d'un excitateur, mais, en approchant davantage, éteint cette même étincelle, le courant, facile à déceler au galvanomètre, continuant d'ailleurs à passer. D'après la théorie précédente, il semblerait qu'en exagérant l'ionisation l'on doit augmenter le débit et l'éclat de l'étincelle : on voit que c'est l'inverse qui a lieu.

On peut citer plusieurs faits analogues : un tube de Geissler dont on chauffe fortement une région cesse d'être lumineux dans cette région; un fil incandescent placé dans ce tube crée autour de lui un espace obscur : or on sait qu'un corps incandescent émet des ions en quantité considérable : sa présence devrait donc exagérer la luminescence du gaz.

Les rayons de Lenard constituent, sans doute possible, un puissant agent d'ionisation; or ils éteignent une étincelle électrique comme

J. de Phys., f“ série, t. VIl. (Mai 1908.) 
le fait un corps radioactif. C'est également par suite de l'action ionisante des rayons cathodiques qu'un tube de Crookes semble parfaitement vide : aucune lumière anodique n'y apparaît, sauf si l'anode est abritée contre les rayons issus de la cathode : la luminescence est totalement supprimée dans l'espace traversé par les corpuscules cathodiques dont le pouvoir ionisant est précisément invoqué pour expliquer la lumière de la décharge. Ce n'est pas l'insuffisance du nombre des molécules qui est la cause de l'obscurité, car il suffit de modifier très peu les conditions de la décharge pour que l'ampoule se remplisse d'une lumière intense (1); on fait également apparaître la lumière anodique ou positive en déviant les rayons cathodiques avec un aimant, c'est-à-dire en les supprimant dans toute une région de l'ampoule.

Dans la phosphorescence de l'oxygène mélangé de beaucoup d'azote ( 50 à $800 / 0$ d'azote), non seulement la production de lumière se fait sans ionisation, car un champ électrique ou magnétique est sans action sur l'établissement et la propagation si singulière de cette phosphorescence, mais la présence d'une cathode en activité fait immédiatement cesser la phosphorescence.

Enfin, les rayons découverts par M. J.-J. Thomson, et qui émanent d'une cathode exactement comme les rayons cathodiques, possèdent la remarquable propriété de ne produire aucune lumière dans l'oxygène pur que les rayons cathodiques illuminent, au contraire, très brillamment.

Ces rayons, qui transportent, comme je l'ai montré, des charges positives, ne se voient que si l'oxygène contient un peu d'hydrogène et n'illuminent que ce dernier gaz.

Il semble résulter de ces faits que, loin d'être une cause de production de lumière, l'ionisation ait plutôt pour effet de rendre la décharge obscure. Dans le cas où des particules chargées produisent de la lumière sur leur trajet (gaine négative), on vient de voir que le signe de leur charge n'est pas indifférent, ce dont ne peut évidemment rendre compte l'hypothèse de l'ionisation par simple choc.

En dehors de ces difficultés qu'elle rencontre indirectement, la théorie précédente conduit à des conséquences immédiates qu'on

(1) Le refoulement du ga\% par les rayons cathodiques ne saurait être admis, car le résultat est le même quand on prend un tube cylindrique droit avec des électrodes placées aux deux bouts. Le gaz devrait alors s'accumuler vers l'anode et la luminosité y serait très vive. Or on n'observe rien de semblable. 
peut aisément soumettre au contrôle de l'expérience : le rôle fondamental qu'elle fait jouer à l'émission cathodique oblige à admettre qu'aux fortes pressions (au-dessus de 1 millimètre de mercure) pour lesquelles'les rayons cathodiques, peu rapides, sont complètement absorbés au voisinage immédiat de la cathode, la lumière doit apparaître en premier lieu à la cathode, et dans la gaine négative qui constitue le premier phénomène visible produit par les rayons cathodiques. La lumière positive ne duit commencer qu'ensuite, et son apparition doit se faire progressivement dans le sens cathodeanode, suivant ainsi la marche du processus d'ionisation commencé à la cathode.

En second lieu, la gaine négative ne saurait être absente d'une décharge électrique, puisqu'elle en marque le début.

On verra dans cet exposé que la luminescence du gaz progresse au contraire dans le sens anode-cathode $\left({ }^{1}\right)$, avec une vitesse n'ayant aucun rapport avec le champ, et pouvant devenir presque infinie pendant que celui-ci tombe presque à zéro; on verra également que la gaine négative peut manquer totalement et que cette suppression caractérise précisément les décharges intenses.

L'émission cathodique, loin d'aider à la formation de l'étincelle, en gênerait donc plutôt le passage.

En présence de cette insuffisance manifeste des théories admises, j'ai entrepris une série d'expériences faites dans des conditions aussi différéntes que possible de celles qu'on adopte habituellement. Aucune raison n'obligeant à choisir, pour expliquer l'étincelle ou l'arc, des pressions très faibles, j'ai le plus souvent opéré entre 1 centimètre de mercure et la pression ordinaire. Dans ces conditions, la lumière ou gaine négative a unefaible épaisseur et la partie prédominante de la décharge est la colonne positive. A ces fortes pressions cette colonne présente des contours suffisamment définis et surtout ne remplit pas les ampoules. Il suffit alors de prendre des tubes très larges, ou des ballons, pour obtenir des résultats indépendants de l'influence des parois. J'ai également utilisé des courants beaucoup plus intenses qu'on ne le fait ordinairement, et le plus souvent compris entre $0^{\mathrm{amp}}, 01$ et $0^{\mathrm{amp}}, 2$.

La 'source employée était en général une bobine d'induction

( $\left.{ }^{1}\right)$ Ce phénomène a été déjà signalé par M. J.-J. Thomson (Experim. Researches) et par M. E. Wiedemann (Wied. Ann., tome LXVI). 
montée en transformateur. Les mesures de voltage et d'intensité peuvent ainsi être faites avec une approximation souvent suffisante, et le débit peut être à la fois notable et régulier. Exceptionnellement . j'ai utilisé des accumulateurs; le plus souvent j'ai réalisé une source continue de faible durée avec un condensateur de 1 à 2 microfarads chargé à 2000 ou 3000 volts par le transformateur muni d'une soupape, et déchargé dans les ampoules au travers d'une résistance ou d'une bobine de réaction, ou même directement. On peut ainsi obtenir une décharge dont on règle à volonté la durée, et on arrive même à l'établissement d'un régime permanent qu'il serait sans intérêt de prolonger.

Les expériences ont èté faites presque sans exception dans l'air à diverses pressions.

LES DEUX FORMES DE LA DÉGHARGE.

Précurseurs de la décharge. - Soit par exemple un large tube ( 3 centimètres) de 80 à 90 centimètres de longueur, contenant de l'air raréfié à 15 millimètres environ et relié au transformateur. Vers 6000 à 8000 volts (efficaces), des lueurs apparaissent, des aigrettes sinueuses courent sur les parois. A 10000 volts, ces décharges se régularisent: des stratifications très nettes se dessinent et occupent toute la section du tube; il y a une faible gaine négative; mais la lumière émise est à peine visible et le courant n'atteint pas un millième d'ampère moyen ; cette intensité est limitée par le tube lui-même et non par les résistances extérieures ; elle ne tend pas à croître spontanément. L'aspect est celui d'un tube dontle gaz est fortement raréfié. A 12000 volts, la décharge se constitue réellement et présente les caractères qui correspondent à la pression de 15 millimètres. Son apparition est brusque et l'intensité s'élève instantanément à un dixième d'ampère environ; le voltage tombe à 7000 volts. Ce sont maintenant les résistances extérieures qui limitent l'intensité.

Phénomène de Geissler. - La décharge une fois constituée, on observe les aspects bien connus (gaine négative et couche lumineuse en contact avec la cathode, espace de Faraday et lumière positive). Aux fortes pressions ( 1 centimètre de mercure et au-dessus), la gaine négative est insignifiante et son rôle n'est pas prépondérant. La colonne positive n'occupe que la région axiale du tube, et son. 
diamètre est de 10 à 20 millimètres, suivant l'intensité du courant ; il augmente avec la raréfaction.

Dans ces conditions, la lumière positive s'allonge toujours lorsqu'on fait croìtre l'intensité (1), et cela dans des proportions énormes.

Ainsi, dans un ballon (pour éviter l'influence des parois), la distance des électrodes étant de 10 à 11 centimètres, et la pression de l'air 15 millimètres, la longueur de la lumière positive varie de quelques millimètres à près de 10 centimètres, quand l'intensité

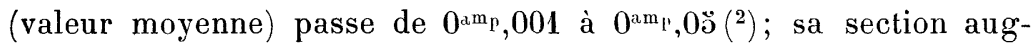
mente en même temps.

L'allongement et l'accroissement de section de la lumière positive s'accompagnent d'une diminution notable de la différence de potentiel aux bornes. Dans le ballon précédent, par exemple, et en laissant passer les deux alternances du courant pour que les indications du voltmètre électrostatique aient un sens défini, le voltage s'abaisse de 1100 volts efficaces à 800 volts quand le courant s'élève de quelques millièmes à quelques centièmes d'ampère. Avec des accumulateurs, le résultat est analogue et les indications fournies par les instruments de mesure sont alors tout à fait exactes.

L'ampoule présente ainsi, aux fortes pressions, une sorte de résistance négative : la chute de tension aux électrodes diminue quand le courantaugmente, etle régime ne peut être stable avec une source à potentiel constant, à moins de mettre en circuit une résistance donnant lieu à une chute de tension croissant avec le courant. L'emploi de cette résistance est inutile quand la source est une bobine montée en transformateur, la résistance (ohmique et inductive) de cet appareil étant considérable. On constate alors simplement que le voltage aux bornes varie en sens inverse de l'intensité.

Aux basses pressions, vers $0^{\mathrm{mm}}, 1$ par exemple, les choses se passent tout autrement: la lumière négative est très développée, entoure généralement toute la cathode; la chute cathodique cesse d'être invariable pour croître avec le courant, et ce phénomène qui, précédemment, n’intervenait pas, régit maintenant presque à lui seul les

(1) Soit en réduisant les résistances extérieures, soit en augmentant la force électromotrice de la sourse, si celte source présente une résistance considérable.

(2) Indications données par un ampèremètre à cadre mobile et à shunt. Comme le courant ne passe que pendant une alternance sur deux. le courant réel est au moins double de celui indiqué ici. 
variations du voltage. Dans ces conditions, le régime est stable même en l'absence de résistances extérieures, car le courant ne peut augmenter que si le voltage s'élève.

La lumière positive, ou colonne anodique, tend toujours à se centrer sur l'axe du tube, même si les électrodes sont décentrées. La répulsion exercée par les parois est assez forte pour masquer complètement l'effet d'entraînement produit par l'air chaud, tant que la pression ne dépasse pas 10 millimètres de m€rcure.

Décharge disruptive. - Arc. - Supposons une ampoule à rayons cathodiques reliée à une forte bouteille de Leyde qu'on peut charger progressivement par une source quelconque; en prenant par exemple les résultats obtenus dans un cas particulier choisi au hasard, on aura un commencement d'émission cathodique à 6000 volts (différence de potentiel vraie, la source qui alimente l'ampoule étant continue) : si on élève le voltage aux bornes de la bouteille, l'émission devient plus intense; mais, entre 6000 et 12000 volts, elle présente toujours ce caractère que le régime est stable et que le courant, extrêmement faible, ne peut augmenter que si la différence de potentiel augmente. Si on abandonne à eux-mêmes l'ampoule et la bouteille, en cessant de fournir de l'électricitéà celle-ci, l'émission cathodique continue pendant un certain temps (trente secondes par exemple), s'affaiblit de plus en plus et s'arrête quand le voltage est revenu à 6000 volts environ. La bouteille de Leyde reste ainsi chargée, l'ampoule n'a fait disparaître que l'excès de charge au-dessus de 6000 volts. Mais, si on atteint 12000 volts, il en est tout autrement : une brillante luminescence apparaît entre l'anode et la cathode, l'ampoule, qui précédemment semblait vide et dont les parois fluorescentes seules émettaient dela lumière, se remplit instantanément de luminosité; en même temps se produit un bruit sec assez fort. Ce phénomène ne dure qu'un temps à peine appréciable et la bouteille de Leyde est aussitôt complètement déchargée. Cette étincelle réellement disruptive a donc joué le rôle d'un véritable court-circuit, et le courant instantané ainsi produit est suffisant pour donner une impulsion notable à l'aiguille d'un ampèremètre thermique de $0^{\mathrm{amp}}, 1$ ou $0^{\mathrm{amp}}, 2$.

L'aspect est en outre entièrement différent de celui qu'on observe d'ordinaire. Il n'y a plus d'émission cathodique, la polarité disparaît et la décharge devient symétrique; aux deux électrodes apparaissent des aigrettes brillantes, colorées par des vapeurs métalliques, et un arc pâle, déviable à l'aimant, réunit ces aigrettes. Il est remar- 
quable que celles-ci ne se forment pas aux points d'où seraient partis des rayons cathodiques.

Le même phénọmène s'obtient à toutes les pressions : on peut par exemple charger un microfarad relié par une clef Morse à deux électrodes disposées, à 10 centimètres de distance, dans un ballon rempli d'air à 10 millimètres. Jusqu'à 2000 volts, la manœuvre de la clef ne donne que la décharge de Geissler dont le débit est faible et la durée très sensible, et le voltage du condensateur ne tombe pas à zéro; à 2500 volts et au-dessus(1), c'est une véritable étincelle qui se produit, accompagnée d'un bruit sec. Un trait de feu, entouré d'une auréole, sans polarité apparente, réunit les deux électrodes et se termine sur chacune d'elles par une forte aigrette colorée à peu près sphérique; la gaine négative et l'espace de Faraday ont disparu. Cette étincelle, de durée inappréciable, décharge entièrement le condensateur et produit dans le circuit des effets inductifs intenses. Le courant instantané prend une valeur considérable et porte facilement à l'incandescence un fil métallique fin $\left({ }^{2}\right)$. Si la distance des électrodes n'est pas trop grande (วั à 6 centimètres pour une pression de 15 millimètres), le passage du phénomène de Geissler à la décharge symétrique sans gaine négative se fait même sans clef Morse, en élevant simplement le débit de la source qui charge le condensateur (transformateur avec soupape), la clef Morse étant supprimée. On obtient ainsi une série d'étincelles'bruyantes, se succédant à des intervalles réguliers qui représentent la durée de charge du condensateur. A chacune de ces étincelles l'aiguille d'un voltmètre statique relié à l'ampoule subit une chute brusque, tandis qu'elle demeure fixe si on maintient la forme ordinaire de décharge.

On facilite beaucoup le passage d'une forme à l'autre en faisant intervenir un champ magnétique transversal.

Dans ces expériences, quand l'étincelle disruptive est précédée par

(1) On verra plus loin que l'élévation du voltage n'est pas directement cause de la transformation de la décharge: elle ne sert qu'à permettre au courant de prendre rapidement une forte intensité.

(2) Si on remplace ce fil par une petite lamps à incandescence à filament droit de quelques centimètres de longueur, on observe un phénomène très singulier : la décharge passe, non par le fil, mais par le gaz. L'introduction d'une résistance inductive faible (moins d'un millième de henry) supprime la luminescence du gaz, mais le courant ne passe que par la surface du filament et produit une lumière jaunâtre instantanée. Ralentissant encore la décharge, le filament est enfin porte à l'incandescence. 
le phénomène de Geissler, le trait de feu évite la gaine négative et aboutit sur la cathode à l'endroit où cette gaine cesse d'exister. Cela résulte de l'incompatibilité de la lumière positive et de la lumière négative, et c'est pour la même raison qu'au vide de Crookes la décharge en arc n'aboutit jamais au point de la cathode qui émet des rayons cathodiques (centre). L'action du champ magnétique s'explique par la déviation qu'il imprime à ces rayons, leur absence facilitant l'allongement de la lumière positive et son arrivée à la cathode.

On ne modifie pas beaucoup l'aspect général de cette décharge en intercalant dans le circuit une bobine de self-induction même importante : le trait de feu et l'auréole cylindrique qui l'enveloppait se mélangent simplement pour former une colonne lumineuse homogène et la hauteur du bruit baisse à mesure que l'impédance augmente. On peut ainsi augmenter dans des proportions énormes la durée de la décharge en la faisant passer dans un enroulement de transformateur à noyau de fer de 1 henry, ce qui, pour 1 microfarad, abaisse la fréquence à 160 . Si la bobine de self-induction est le primaire d'une bobine de Ruhmkorff, le secondaire de celle-ci donne d'aussi belles étincelles qu'avec le montage ordinaire.

L'intercalation d'une résistance produit un autre effet : l'intensité peut être considérablement réduite et la durée de la décharge augmente alors beaucoup. La polarité apparaît progressivement à mesure que la résistance augmente, et bientôt le trait de feu cesse d'atteindre la cathode. La gaine négative se montre à nouveau et on est revenu au phénomène de Geissler. Il en serait certainement de même avec une self-induction suffisamment forte, produisant une résistance apparente de quelques milliers d'ohms. On voit ainsi que l'étincelle disruptive n'est pas autre chose que la décharge réduite à la colonne anodique ou lumière positive.

Les fig. 2 et 3 représentent quelques décharges obtenues dans ces conditions. La fig. 1 indique le montage de l'expérience.

La $f g .3$, correspondant à une distance d'électrodes mieux appropriée au voltage disponible, montre nettement la suppression de la gaine négative lors de l'établissement de l'arc.

Il est facile de s'assurer que la forme disruptive de la décharge est identique à l'arc électrique : il suffit de remplacer le condensateur par une batterie d'accumulateurs dont la capacité est pour ainsi dire infinie; $ّ 00$ volts suffisent si la distance des électrodes est réduite 
à 15 millimètres environ( $\left.{ }^{1}\right)$. Un rhéostat convenable règle l'intensité. Dans ces conditions, jusqu'à $0^{\mathrm{amp}}, 15$, on a la décharge ordinaire, avec gaine négative de plus en plus intense; le voltage aux électrodes diminue à mesure que l'intensité augmente, mais reste voisin de 400 volts, c'est-à-dire supérieur à la chute cathodique; quand le courant atteint $0^{\mathrm{am}}, \mathbf{1}$ ๖े, l'espace de Faraday et la gaine négative disparaissent instantanément, et le courant prend sensiblement la même valeur que si l'ampoule était mise en courtcircuit $\left(1^{\mathrm{amp}}, \stackrel{\circ}{\mathrm{g}}\right.$ dans cette expérience). Le voltage aux électrodes est insignifiant, la chute cathodique a disparu avec la gaine négative, et l'aspect est exactement celui de l'arc électrique ordinaire.
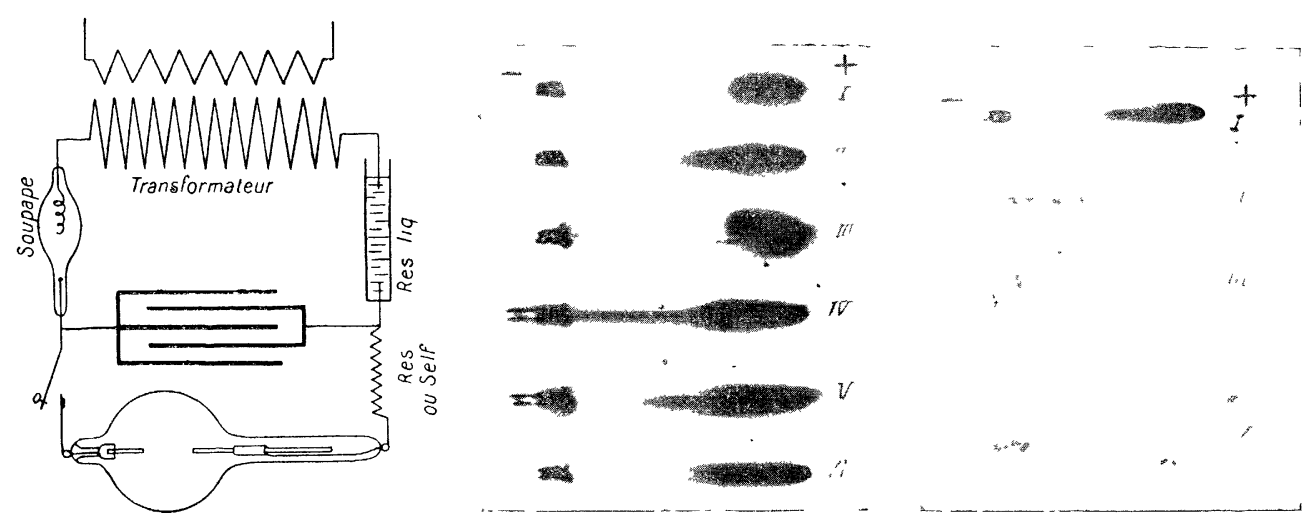

FIG. 1, 2 et 3.

Fiı. 1. - Montage de l'expérıence. - Condensateur de 1 à 2 unicrofarads relié à un voltmètre tatique (non figuré); ballon de 15 centinètres de diamètre ; électrodes d'aluminium de $2^{\mathrm{mm}}, \bar{\partial}$, dont l'une mobile dans une coulisse (soutenue par un tube de verre non figuré); pression, lis millimètres.

Fiø. 2. - Distance des électrodes, 10 centimètres. - I, II, III et IV, décharæes sans résıstiunce ni self; voltages de charge croissant de 2000 à 3000 volts. - V et VI, décharges à 3000 volts avec résistances de 3000 et 5000 ohms en série.

Fig. 3. - Distance des électrorles, 7 centimètres. - I, ?000 volts sans résistance. - II et III, 3000 volts sans résistance: disparition totale de la gaine négative. - IV, 3000 volts arec résistance de $200 \mathrm{ohms}$. $-\mathrm{V}, 3000$ volts et 5000 ohms; réapparition de la gaine négative.

Ce qu'on observait avec le condensateur relié d'une manière permanente à l'ampoule ne différait de ce qui précède que par l'absence de durée de l'arc. Le phénomène est donc bien le même dans les deux cas.

(1) Avec ce faible voltage on est toutefois obligé d'amorcer la décharge en intercalant dans le circuit le secondaire d'un transformateur qu'on met en artivité pendant une ou deux secondes : on met ensuite en court-circuit ce secondaire, ce qui le supprime sans interrompre aucune communication. 
Dans ces expériences faites à de fortes pressions, le voltage aux électrodes diminue à mesure qu'on approche du passage de la première forme de décharge à la seconde. Au vide de Crookes, l'inverse avait lieu. Mais, dans l'un et l'autre cas, c'est toujours par accroissement de l'intensité qu'on obtient l'étincelle disruptive ou l'arc. Il est donc naturel d'admettre que c'est de cette intensité que dépend la transformation; quant au voltage, il monte ou descend suivant les lois indiquées au commencement de ce chapitre.

Ainsi l'étincelle disruptive oscillante et l'arc électrique sont des décharges réduites à la lumière positive réunissant les deux électrodes, et dont la résistance varie en sens inverse de l'intensité. L'absence de chute cathodique, précisément caractéristique de l'arc, est commune aux deux phénomènes et s'explique par la suppression de la gaine négative.

La décharge qu'on observe dans un tube sans électrodes est en général réduite à la lumière positive, c'est un arc. Cette absence de différenciation est très facile à observer avec la vapeur d'iode, qui donne deux spectres très différents pour la lumière positive et la lumière négative.

LES RAYONS CATHODIQUES ET L'ESPACE OBSCUR DE FARADAY.

L'étude des deux formes de la décharge semble montrer que la gaine négative empêche la lumière positive d'atteindre la cathode. Cela paraît presque évident quand on voit la lumière positive contourner la gaine pour atteindre les régions nues de la cathode en allongeant son trajet de 1 ou 2 centimètres. On est ainsi conduit à considérer l'émission cathodique et l'ionisation qu'elle détermine dans le gaz comme un obstacle à la formation de la colonne anodique. L'espace obscur de Faraday s'explique alors sans difficulté: il résulte de ce que la lumière positive est supprimée dans toute la région intéressée par les rayons cathodiques. Cet espace doit en conséquence augmenter d'étendue avec la raréfaction et se raccourcir si on écarte, avec un aimant, les rayons cathodiques : or c'est en effet ce qu'on observe.

$\mathrm{Si}$, aux fortes pressions, on augmente l'intensité du courant, la gaine négative s'étend de plus en plus, mais l'émission cathodique par unité de surface varie peu, la chute cathodique reste constante, le pouvoir pénétrant des rayons et leur nombre dans la direction de 
l'anode demeurent invariables; la chaîne anodique, devenue plus intense, peut lutter avantageusement contre la destruction par le choc des corpuscules, et elle s'allonge, réduisant ainsi l'étendue de l'espace de Faraday; quand celui-ci est supprimé, l'arc s'établit.

Aux basses pressions, la chute cathodique croît avec le courant, le pouvoir pénétrant des rayons augmente et la lumière positive rétrograde vers l'anode. L'établissement de l'arc est alors moins facile à comprendre, à moins d'admettre qu'à la faveur de la discontinuité de l'émission cathodique il n'ait le temps de se former entre deux trains de corpuscules. Quoi qu'il en soit, cet arc n'atteint jamais la cathode aux points d'où on voit partir des rayons cathodiques.

On peut vérifier directement que les corpuscules cathodiques suppriment la lumière positive partout où ils la rencontrent : il suffit de disposer les choses comme l'indique la fig. 4. Les électrodes A

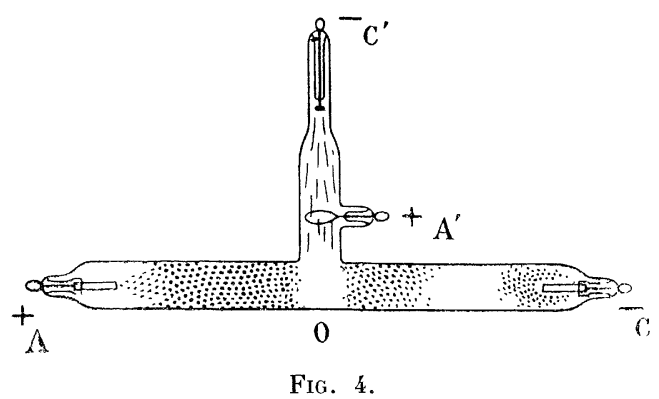

et $\mathrm{C}$ sont reliées à une première source (transformateur); les électrodes $\mathrm{A}^{\prime}$ et $\mathrm{C}^{\prime}$ reçoivent le courant d'un autre transformateur ou d'une bobine à trembleur synchrone. La cathode C' est placée dans un tube étroit, de manière à donner des rayons énergiques même à une pression relativement forte. Le courant étant établi entre $\mathrm{A}$ et $\mathrm{C}$, dès que $\mathrm{C}^{\prime}$ entre en activité, un espace obscur apparaît en $\mathrm{O}$. Si on approche un aimant du faisceau émis par $\mathrm{C}^{\prime}$, cet espace se comble immédiatement.

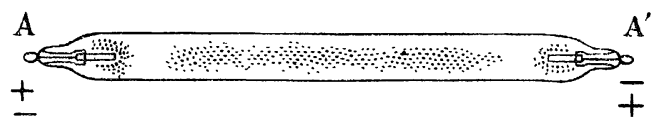

Fig. 5.

On peut réaliser l'expérience autrement: un tube droit $\mathrm{AA}^{\prime}(f g$. à) 
est alimenté par une source alternative, sans soupape. Par suite de l'échange des polarités des électrodes, l'espace de Faraday correspondant aux alternances paires devrait paraître rempli par la colonne anodique des alternances impaires, l'effet de superposition ne pouvant disparaître que si l'on fait usage d'un miroir tournant. Or, pour une pression convenable, on voit au contraire deux espaces de Faraday parfaitement obscurs, et il n'y a de lumière positive que dans la région médiane du tube.

L'explication est immédiate. Près de chaque électrode, l'émission cathodique produit alternativement une ionisation intense qui subsiste quelque temps. Il suffit que ce temps soit supérieur à une demipériode pour empêcher la formation de la lumière positive. En abaissant la pression, les ions se diffusent plus vite et le phénomène disparaît. Si on approche un aimant, on dévie les rayons cathodiques et l'espace obscur se comble aussitôt.

Ainsi, dans l'espace de Faraday et la région circumcathodique, le courant passe uniquement par convection (bombardement et ionisation) comme entre deux plateaux dans l'air soumis aux rayons $\mathrm{X}$; dans la lumière positive, le transport de l'électricité serait analogue à la conduction. On peut donc comparer un tube de Geissler à une résistance métallique en série avec un appareil à grêle de Volta. L'établissement de l'arc consisterait à supprimer l'appareil à grêle et à réunir directement les électrodes par un conducteur, d'où la possibilité d'obtenir des oscillations.

DÉVIATION MAgNétiQue de La LuMière POSITIVE.

Considérons un large tube contenant de l'air assez peu raréfié (pression supérieure à 1 centimètre) pour que la lumière positive n'occupe que la région axiale et offre l'aspect d'un cordon lumineux de la grosseur d'un crayon. L'apparence rectiligne de ce cordon, contrastant avec la forme d'arc qu'on obtient dans un vaste ballon, nous apprend que les parois du tube exercent sur la lumière positive une répulsion capable de compenser l'entraînement dû à l'air échauffé par la décharge (1). Une autre preuve de l'existence de cette répulsion est fournie par ce fait que, si les électrodes sont hors de

(1) Si la pression est très supérieure à 1 centimètre de mercure, par exemple triple de cette valeur, l'action des courants d'air chaud devient prédominante. 
l'axe du tube, la lumière positive se centre néanmoins sur cet axe. Il y a donc lieu de tenir compte de cette répulsion dans l'interprétation de tous les résultats relatifs à des déviations d'origine quelconque.

Si maintenant nous mettons le tube entre les branches d'un aimant en fer à cheval, nous obtiendrons à peu près la déformation indiquée sur la $f g .6$. On remarque qu'au point $M$, où l'écart est maximum, la tangente est parallèle à la direction initiale; autrement

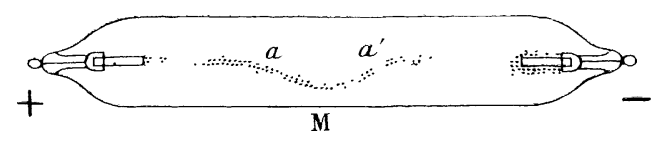

FIG. 6.

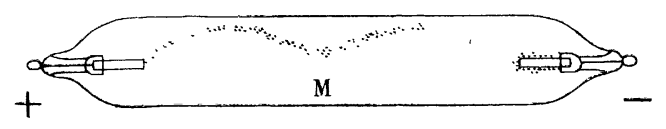

FIG. 7.

dit, la déviation est nulle au point où le champ déviant est maximum. L'hypothèse d'une convection électrique par des ions ou particules électrisées quelconques ne paraît pas susceptible d'expliquer ce simple fait : il serait surprenant que, quelles que soient la largeur du tube, la raréfaction, et la valeur du champ, la résultante de toutes ces actions soit toujours nulle exactement au point où le champ est maximum; il n'est d'ailleurs pas difficile de remplacer l'action directrice des parois par une force de nature différente ne suivant pas les mêmes lois; il suffit d'élever la pression à こ̃ centimètres, la convection par l'air chaud élève la lumière positive vers le haut du tube et, dans ce cas encore, l'aimant donne le même résultat $(f g .7)$. Remarquons aussi qu'en $a$ et $a^{\prime}(f g .6)$ il y a deux points d'inflexion. La courbure de la lumière positive change donc deux fois de signe dans un champ magnétique de sens constant.

Il est très facile d'expliquer ces effets en imaginant que la colonne anodique est une chaîne extensible pourvue de cohésion, tendue entre l'anode et la cathode, et se comportant comme un conducteur ordinaire. La figure d'équilibre en présence d'un aimant est alors aisée à prévoir, c'est celle d'un fil repoussé par les parois et qu'on écarterait de sa position, en un point, au moyen d'une poulie solli- 
citée normalement à la direction de l'axe du tube. On aura les deux inflexions $a$ et $a^{\prime}$ et, au point le plus dévié, la tangente sera parallèle à la direction initiale. On arrive à la même conclusion en répétant l'expérience classique de De la Rive avec un courant plus intense et plus continu que les décharges d'une bobine de Ruhmkorff ordinaire. Quelques centièmes d'ampère en courant alternatif avec soupape conviennent parfaitement; on voit alors la lumière positive s'enrouler en hélice autour du barreau de fer qui prolonge le noyau de l'électro-aimant. On obtient un enroulement tout à fait semblable et de même sens avec un fil souple parcouru par un courant et placé auprès d'un électro-aimant analogue au précédent.

Dans cette expérience, il est indispensable que l'une des extrémités du conducteur flexible. soit fixe. Il suffit pour cela d'attacher une pointe à l'une des électrodes circulaires de l'œuf électrique.

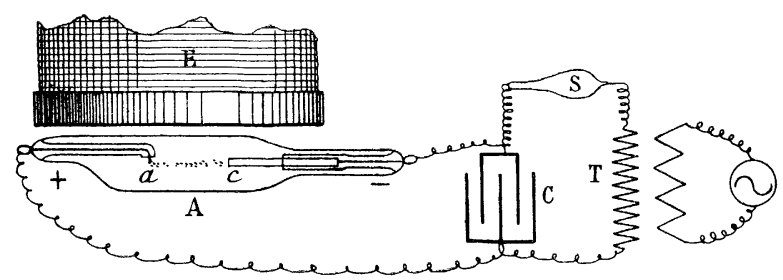

FIG. 8 ,

11 convient évidemment d'étudier la déviation dans un champ à peu près uniforme et de manière à obtenir un enroulement plan. L'emploi d'un ballon, de préférence plat, supprime l'influence des parois ; l'anode doit être un fil perpendiculaire au plan de l'enroulement ('). La source électrique est soit un transformateur, soit un condensateur de 1 ou 2 microfarads; dans le premier cas, l'intensité, variable pendant toute la durée de la décharge, donne une nappe plane limitée par deux courbes; dans le second cas, le courant se maintient constant pendant presque toute sa durée, d'ailleurs très faible, et on obtient une bande lumineuse étroite préférable pour les mesures.

(1) Si on dispose l'anode et la cathode dans le prolongement l'une de l'autre, le champ, tendant à faire embrasser le plus grand flux possible par la colonne anodique enroulée, déplace le point d'attache de celle-ci sur l'anode et le fait rétrograder jusqu'à l'endroit où l'anode est soudée à l'ampoule. 
La fig. 9 est la reproduction photographique d'une expérience de ce genre. On voit qu'il s'est produit deux décharges distinctes correspondant à ce qui a été décrit à propos de la fig. 1. L'étincelle disruptive est précédée d'une décharge peu intense et ne s'est établie que quand cette dernière a acquis une certaine intensité.

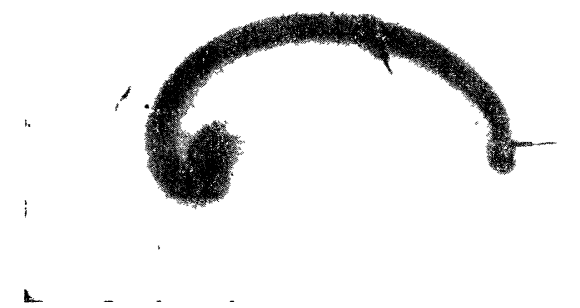

Fig. 9. - Enroulement de la lumière positive dans un champ de 500 unités. Distance des électrodes, 5 centimètres (décharge condensée sans gaine négative). La fig. 8 indique le montage de l'expérience.

La forme générale de l'enroulement est celle d'une spirale. On peut démontrer qu'il en doit être sensiblement ainsi.

La colonne anodique fixée à l'anode et entourée de gaz ionisé se comporte comme un fil métallique plongé dans un milieu sensiblement conducteur; le courant décroît d'une extrémité à l'autre par suite de la déperdition latérale. Admettons que l'intensité varie en raison inverse de la longueur de l'arc compté à partir d'une origine convenable, et supposons constante la tension mécanique du conducteur gazeux. I.e champ magnétique exerce sur chaque élément de ce conducteur une action normale proportionnelle à l'élément considéré et à l'intensité du courant.

L'équilibre a lieu lorsque le rayon de courbure est en chaque point inversement proportionnel à la force, c'est-à-dire au courant puisque le champ est le même partout. Ce rayon de courbure est donc proportionnel à l'arc. Or c'est précisément là une propriété de la spirale logarithmique (l'arc étant mesuréà partir du point asymptotique).

Tout cela est aisé à établir.

Prenons, en coordonnées polaires, l'anode pour origine. Admettons que le long de l'arc $s$ l'intensité I varie suivant la loi :

$$
\mathrm{I}=\mathrm{I}_{0} e^{-m_{\omega}} \text {. }
$$


La force $\mathbf{F}$ exercée par un champ uniforme $\mathbf{H}$ sur un élément de courant et rapportée à l'unité de longueur est :

$$
\mathrm{F}=\mathrm{HI}_{0} e^{-m \omega}
$$

le rayon de courbure $\mathrm{R}$, la tension $\mathrm{T}$ (supposée constante) et la force $\mathbf{F}$ sont reliés par la relation :

d'où :

$$
\mathrm{R}=\frac{\mathrm{T}}{\mathrm{F}}
$$

Posons :

$$
\mathbf{R}=\frac{\mathrm{T}}{\mathrm{HI}_{0}} \times e^{m \omega}
$$

il vient :

$$
\frac{\mathrm{T}}{\mathrm{HI}_{0}}=a \sqrt{1+m^{2}}
$$

$$
\mathrm{R}=a \sqrt{1+m^{2}} e^{m \omega},
$$

qui est le rayon de courbure de la spirale,

$$
\rho=a e^{m \omega} \text {. }
$$

D'autre part, l'arc $s$, compté à partir du point asymptotique, est donné par l'expression :

$$
s=\frac{a \sqrt{1+m^{2}}}{m} e^{m \omega}=\frac{\mathrm{R}}{m}\left({ }^{1}\right) ;
$$

il est bien proportionnel au rayon de courbure.

Si maintenant on remplace $R$ par sa valeur donnée plus haut, il vient :

$$
\mathrm{I} s=\frac{\mathrm{T}}{m \mathrm{H}}=\text { constante. }
$$

(1) On a en effet:

et comme

il vient

$$
\begin{gathered}
d s^{2}=\rho^{2} d \omega^{2}+d \rho^{2}, \\
d_{\rho}=m \rho d \omega, \\
d s^{2}=\rho^{2}\left(1+m^{2}\right) d \omega^{2},
\end{gathered}
$$

d'où, en remplaçant $\rho$ par sa valeur $a e^{m \omega}$ :

$$
s-s_{0}=\frac{a \sqrt{1+m^{2}}}{m} e^{m \omega}
$$

si l'arc est compté à partir du point asymptotique, il est nul pour $\omega=-\infty$, et l'expression précédente se réduit ì :

Comme, d'autre part, on a :

$$
s=\frac{a \sqrt{1+m^{2}}}{m} e^{m \omega}=\rho \frac{\sqrt{1+m^{2}}}{m} .
$$

$$
\frac{d s}{d \omega}=\mathrm{R}, \quad \text { il suit de là que } \quad s=\frac{\mathrm{R}}{m} .
$$


L'intensité varie donc bien en raison inverse de l'arc, quand l'enroulement se fait en spirale, et réciproquement.

Si on connaît les valeurs $I_{1}$ et $I_{2}$ du courant aux deux extrémités de la colonne anodique, les valeurs correspondantes $s_{1}$ et $s_{2}$ de l'arc fixent la position de cette colonne sur la spirale, et la différence des arguments $\omega_{1}$ et $\omega_{2}$ indiquera le nombre de spires de l'enroulement.

Remarquons que plus I augmente, plus la valeur correspondante de $s$ diminue, et plus, par conséquent, on se rapproche du point asymptotique. On voit ainsi qu'augmenter l'intensité de la décharge revient à faire glisser la colonne anodique le long de la spirale géométrique; elle semblera ainsi s'enrouler autour de l'anode comme un ressort de montre qu'on remonte.

La dernière égalité montre encore qu'il est équivalent de faire varier I ou H. Donc, en augmentant l'intensité du champ, on obtiendra le même résultat qu'en faisant croître le courant.

L'expérience vérifie entièrement ces conclusions.

Le sens dans lequel se fait l'enroulement n'est pas moins important que sa forme ; il est régi par la loi suivante :

Pour un observateur qui regarde dans la direction du champ, l'enroulement est décrit à partir de l'anode dans le sens des aiguilles d'une montre; autrement dit il est dextrorsum.

$\mathrm{Si}$ on avait affaire à des charges positives libres, les trajectoires de ces charges seraient au contraire décrites dans le sens inverse de celui des aiguilles d'une montre (').

Des charges négatives suivraient, il est vrai, des trajectoires dextrorsum, mais elles les décriraient dans le sens cathode-anode. $\mathrm{Si}$ on les considère à partir de l'anode, le sens est sinistrorsum.

Ainsi l'hypothèse d'ions positifs ou négatifs en mouvement conduit à un sens d'enroulement exactement inverse de celui qu'on observe ; cette hypothèse est donc à rejeter; on est en présence de l'équilibre d'un fil et non de la trajectoire d'une masse $\left({ }^{2}\right)$ et le problème appartient au domaine de la Statique et non à celui de la Dynamique.

Cette opposition peut se mettre sous une autre forme et s'énoncer comme il suit :

La trajectoire décrite par une masse électrisée en mouvement dans

(1) On verra que la décharge part de l'anode: il y avait donc lieu de considérer des particules positives capables, comme celles du radium, de parcourir un grand espace dans l'air.

Les ions ordınaires, même ceux des flammes, dont la mobilité atteint 80 centimètres pour les ions positıls, seraient tellement sensibles au champ magnétique qu'ils ne pourraient s'élolgner de l'anode dans l'expérience de la fig. 9.

(2) De même la courbure de la parabole décrite par un projectile pesant est inverse de celle d'un fil pesant fixé en deux points (chaînette).

J. de Phys., $4^{\mathrm{e}}$ série, t. VII. (Mai 1908.). 
un champ magnétique est parcourue dans un sens tel que le courant de convection produit diminue le champ magnétique.

Un conducteur parcouru par un courant et placé dans un champ s'enroule au contraire de manière à augmenter ce champ.

Or ce dernier cas est celui de la lumière positive. Celle-ci doit donc être considérée comme résultant du passage d'un véritable courant de conduction dans une chaîne de particules gazeuses immobiles ou à peu près, tendue entre l'anode et l'espace de Faraday $\left(\mathbf{1}^{\mathrm{er}}\right.$ mode de décharge) ou entre l'anode et la cathode ( $2^{\mathrm{e}}$ mode $)$.

Particularités de la déviation. - Quand on établit d'abord le champ, et qu'on fait ensuite passer la décharge, la lumière positive apparaît toute déviée. Il n'y aurait là aucune difficulté dans l'hypothèse balistique, une trajectoire étant complètement définie par les conditions initiales, c'est-à-dire avant d'ètre parcourue. Mais, cette hypothèse étant inadmissible et la colonne anodique ayant les propriétés d'un conducteur parcouru par un courant, il semble que la déviation devrait partir de zéro; on s'attendrait ainsi à voir la colonne anodique, d'abord rectiligne, se déplacer rapidement jusqu'à sa position d'équilibre et balayer ainsi une aire. Ce régime variable se répétant avec un courant intermittent 40 ou 50 fois par seconde, cette aire serait extrêmement visible: or on n'observe rien de semblable( $\left.{ }^{1}\right)$.

L'explication de cette anomalie est entièrement comprise dans la comparaison suivante; imaginons qu'un fil de platine soit légèrement tendu dans un champ magnétique et qu'on fasse passer dans ce fil un courant capable de le porter au rouge en régime permanent : l'incandescence s'établit lentement, l'inertie calorifique l'emporte sur l'inertie mécanique et le fil est complètement dévié avant de rougir, c'est-à-dire avant d'être visible par sa lumière propre.

Il en est de même avec la lumière positive. Le courant peut parfaitement passer dans la chaîne anodique sans pour cela la rendre immédiatement incandescente. On en a la preuve en répétant simplement l'expérience de De La Rive; avec un courant convenable $\left(0^{\mathrm{amp}}, 04\right.$, valeur moyenne rapportée à une alternance admise par la soupape), on obtient vers 2 centimètres de pression un beau ruban

(1) Si le champ est intense (200 unités au moins), la lumière positive est à la fois déviée et étalée, mais la nappe lumineuse qu'elle donne est limitée non par une droite et une courbe, mais par deux courbes correspondant à des déviations différentes (courants d'intensités différentes). Avec la décharge d'un microfarad, cet étalement même disparaît comme le montre la fig. 9 . 
lumineux qui tourne régulièrement autour du barreau de fer aimanté. Si la rotation est rapide, l'aspect est celui d'une surface de révolution

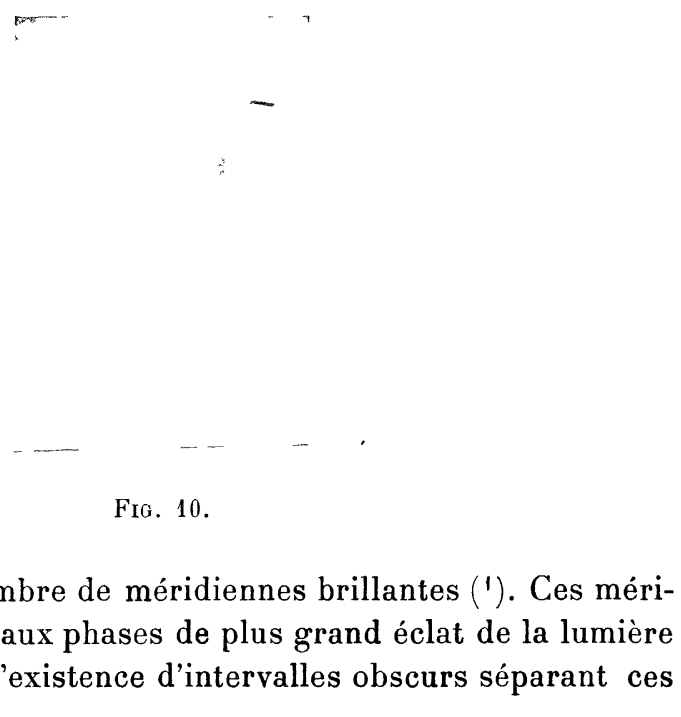

réduite à un certain nombre de méridiennes brillantes (1). Ces méridiennes correspondent aux phases de plus grand éclat de la lumière positive tournante $\left({ }^{2}\right)$. L'existence d'intervalles obscurs séparant ces

(1) Ces lignes lumineuses sont presque toujours tordues en hélice, parce que le champ qui les entraîne n'a pas la même valeur tout le long du barreau. Il y a retard pour l'extrémité située loin de la bobine magnétisante, et cela quel que soit le sens du courant; il n'y a pas à proprement parler enroulement électrodynamique, mais simplement retard mécanique par résistance du mílieu.

(2) Pour une valeur convenable du champ, la durée d'un tour correspond à un nombre exact de périodes et le phénomène paraît fixe. On a, à ce moment, un

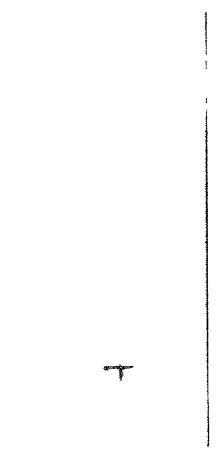

FIG. 11.

moteur synchrone multipolaire à inducteur fixe et à induit gazeux mobile, avec stroboscopie automatique (fig. 11, photographie posée). 
méridiennes prouve que la chaîne anodique a pu tourner sans être visible ; comme la rotation suppose le passage du courant, la conclusion est évidente. C'est ce phénomène que représente la fig. 10.

Reprenons maintenant la précédente comparaison et supposons qu'on fasse passer dans le fil de platine la décharge d'une bouteille de Leyde : le courant atteindra une valeur énormément supérieure à celle qui suffirait à rougir le fil en régime permanent, mais sa durée sera extrêmemen courte; l'inertie mécanique l'emportera cette fois sur l'inertie calorifique; le fil sera porté au rouge avant d'avoir été dévié d'une manière appréciable, et il semblera ainsi que cette décharge est insensible au champ magnétique ( $\left.{ }^{1}\right)$.

Ce qu'on observe avec la lumière positive est tout à fait semblable; la décharge d'une bouteille de Leyde est à peine déviable; celle d'un

(1) Le problème peut être traité d'une manière très simple en supposant un fil rectiligne non pesant et entièrement libre, mais pourvu d'inertie. En courant continu d'intensité $i$, le régime variable est de durée négligeable vis-à-vis du temps nécessaire pour rougir le fil (au moins $\frac{2}{10}$ de seconde pour un filament de lampe), et la force exercée par le champ sur le fil est constante, ainsi que l'accélération correspondante $\gamma$

Le déplacement pendant le temps $t$ nécessaire pour produire l'incandescence est :

$$
\delta=\frac{1}{2} \gamma t^{2}
$$

Dans le ças du condensateur, l'expérience montre $(f g .9)$ que le courant est pratiquement constant pendant la durée de la décharge. Supposons les choses arrangées de telle sorte qu'il en soit de même pour le fil considéré ici. Soit $i^{\prime}$ le courant, $t^{\prime}$ sa durée et $\gamma^{\prime}$ l'accélération; le déplacement sera :

$$
\delta^{\prime}=\frac{1}{2} \gamma^{\prime} t^{\prime 2} \text {. }
$$

Mais les accélérations sont entre elles dans le même rapport que les courants. Il en résulte que

$$
\frac{\delta^{\prime}}{\delta}=\frac{i^{\prime}}{i} \frac{t^{\prime 2}}{t^{2}}
$$

D'autre part, en négligeant la déperdition par rayonnement, on a :

d'où :

$$
\mathrm{AR} i^{2} t=\mathbf{A R} i^{\prime 2} t^{\prime}
$$

$$
\frac{t^{\prime}}{t}=\frac{i^{2}}{i^{\prime 2}}
$$

Le rapport des déplacements est donc:

$$
\frac{\delta^{\prime}}{\delta}=\frac{i^{3}}{i^{\prime 3}}=\sqrt{\frac{t^{\prime 3}}{t^{3}}} .
$$

Les écarts sont en raison inverse des cubes des intensités ou proportionnels aux puissances $\frac{3}{2}$ des durées de décharge. 


\section{PASSAGE DE L'ÉLEGTRIGITÉ DANS LES GAZ 345}

microfarad l'est notablement, sa durée et son intensité étant plus grandes, et la déviation devient très forte si on ralentit la décharge au moyen d'une résistance inductive.

actions électrostatiques.

Si la lumière positive résulte de l'incandescence d'un conducteur gazeux fixe, les divers points de ce conducteur sont à des potentiels différents et, si on rapproche deux de ces points, il doit y avoir attraction. L'expérience est très facile à réaliser avec un tube en $U$ à branches rapprochées. Le courant doit être faible et la chute de potentiel aussi grande que possible. On réussit très bien avec l'interrupteur Wehnelt ou avec l'alternateur de $500 \sim$ du Laboratoire de l'Ecole normale; cette fréquence élevée permet d'obtenir facilement de hauts voltages et d'opérer par suite à de fortes pressions (̋̀ à 8 centimètres). Dans ces conditions, l'action des parois devient insignifiante.

Le résultat est alors le suivant : pour des courants très faibles $\left(0^{\mathrm{amp}}, 001\right.$ à $0^{\mathrm{am}}, 004$ moyen $)$, l'action électrodynamique qui tend à

$$
\text { FIL. } 12 .
$$

ouvrir la boucle en U formée par là chaîne anodique est faible, comparée à l'action électrostatique, et il y a attraction; quand le courant augmente, la répulsion des deux courants parallèles et de sens contraire compense de plus en plus l'attraction et finit par prédominer vers $0^{\mathrm{amp}}, \mathbf{1}(f g .12)$.

On a une représentation exacte du phénomène avec un fil de soie mouillé suspendu en forme d'U aux bornes du transformateur. Suivant l'intensité du courant, on a une attraction ou une répulsion des deux brins du fil. 
Un conducteur suspendu à une anode doit nécessairement présenter une électrisation positive. Il en est de même de la chaìne anodique. Des expériences faciles à imaginer permettent de vérifier que cette chaîne est attirée par un objet chargé négativement, mais le phénomène est particulièrement frappant avec le courantà $500 \sim$. Tout se passe, surtout aux fortes pressions, comme si le tube de verre n'existait pas, et il suffit d'approcher la main à 8 ou 10 centimètres du tube pour attirer la colonne positive, comme on le ferait de la feuille d'or d'un électroscope à cage de verre. Or la main est toujours cathode, et le résultat est d'ailleurs sensiblement le même en approchant du tube une plaque métallique reliée au pôle de la source qui alimente la cathode par l'intermédiaire de la soupape. De cette manière le potentiel de la plaque est aussi bas que possible et la charge alternative de la plaque n'est pas en cause, car elle est nécessairement cathode pendant l'alternance utilisée ( $\left.{ }^{1}\right)$.

CHRONOPHOTOGRAPHIE DE LA DÉCHARGE.

Le dispositif expérimental constamment employé a été le suivant : un objectif extra-lumineux (ouverture $\frac{f}{4}$ environ), adapté à une petite chambre noire, donnait une image réelle de l'étincelle. Près du plan focal conjugué était disposée une large fente encadrant l'image; dans ce plan focal pouvait glisser une plaque sensible placée dans un châssis à coulisse : le déplacement se faisait à la main. Dans le cas où l'ampoule était alimentée directement par un transformateur, l'intervalle de deux images successives donnait automatiquement la mesure du temps, la fréquence du courant étant exactement connue (42 périodes par seconde).

La fig. 13 montre le résultat obtenu quand la décharge se produit dans un ballon rempli d'air à la pression de 10 millimètres, la distance des électrodes étant de 11 centimètres. Une soupape étant intercalée dans le circuit, les images se succèdent à une période d'intervalle $\left(\frac{1}{42}\right.$ de seconde).

(1) Si on relie la plaque à la cathode du tube, après la soupape par conséquent, son potentiel est un peu moins bas. Sa charge n'en est d'ailleurs pas moins alternative, une soupape láissant toujours passer, dans le sens où elle réagit, le courant qui provoque sa réaction, et qui suffit pour charger une capacité aussi petite que celle d'une plaque métallique d'un demi-décimètre carré. 
Si l'on imagine une fente étroite perpendiculaire à l'axe des temps et posée sur l'épreuve, la bande ainsi découpée représente la lumière positive à un instant donné. En déplaçant cette fente parallèlement

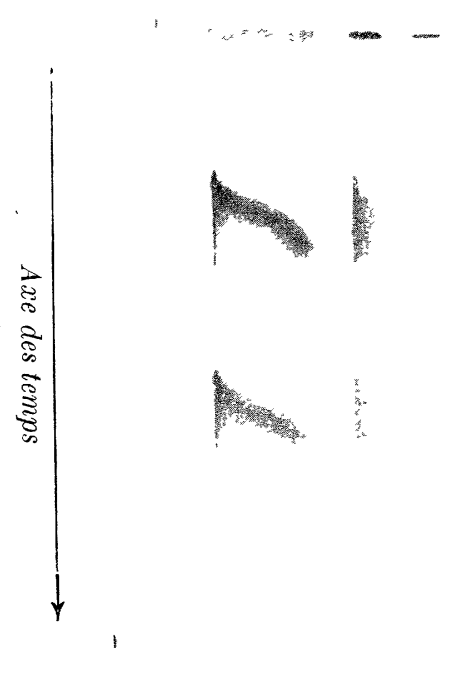

Fig. 13. - Chronophotographie montrant que la lumière positive part de l'anode. Electrodes distantes de 11 centimètres dans un ballon rempli d'air à 15 millimètres.

La décharge est d'abord photographiée sur la plaque au repos (image supérieure). Cette partie de la plaque est ensuite masquée par le volet du châssis et ce dernier, ramené à un bout de la coulisse, est mis en mouvement et passe en vitesse devant l'objectif. L'inconvénient de la courbure imprimée à la lumière positive par l'air chaud est évité en plaçant l'appareil photographique verticalement au-dessus du ballon; on a ainsi une projection horizontale rectiligne de la décharge.

Le temps croît de haut en bas sur la figure. Les décharges se succèdent à $\frac{1}{42}$ de seconde d'intervalle (courant alternatif avec soupape).

à elle-même, on voit immédiatement que la lumière positive part de l'anode, s'allonge lentement (vitesse, $6^{\mathrm{m}}, 7$ par seconde), et que la tête de cette colonne lumineuse est beaucoup plus brillante que le reste. Avec un objectif moins lumineux, l'image se réduirait à une bande inclinée, comme si le phénomène consistait simplement dans le déplacement d'une nébulosité lumineuse partant de l'anode comme 
un projectile. D'autres expériences ont montré que ce surcroît d'éclat du front de la colonne anodique tient à ce que cette partie est en présence de gaz neuf, non encore ionisé ou modifié.

Quant à la gaine négative, elle s'allonge, puis se raccourcit, le long de l'électrode, sa longueur suivant très sensiblement les variations d'intensité du courant pendant la décharge.
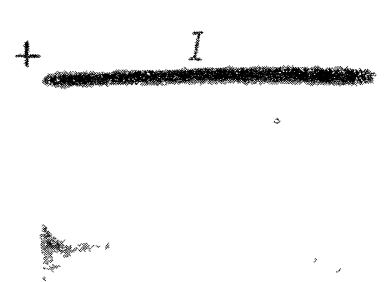

Fia. 14 - Chronophotographies de la décharge dans un long tube, de 5 centımètres de diamètre, à la pression de 15 millimètres. - Distance des électrodes, 47 centimètres. Voltage efficace, 4000 volts ; à gauche, courant faible $(0,04 \mathrm{am}-$ père moyen); à droite, courant plus intense.

La comparaison avec la fig. 13 montre que la vitesse de progression de la décharge est ici beaucoup plus grande.

En haut, photographies sur plaque gu repos, au dessous sur plaque en mouvement; distance de deux points homologues des images, $\frac{1}{42}$ de seconde.

Si on remplace le ballon par un long tube, le voltage augmente à peu près comme la distance des électrodes, et le champ varie à peine. Avec le même courant que précédemment, les vitesses deviennent cependant beaucoup plus grandes. S'il s'agissait d'ions, 
cette vitesse serait au contraire constante pour un même champ et une même pression (fig. 14).

L'emploi du tube fait apparaître un autre phénomène, tout à fait singulier ; la colonne positive tend à se décomposer en plusieurs segments qui commencent en même temps par le côté anodique et dans lesquels la luminosité se déplace encore avec des vitesses très faibles dans le sens anode-cathode.

La fig. 14 montre nettement cette tendance, qui ne se manifeste d'ailleurs qu'au-dessus d'une certaine intensité. On voit de plus, par l'écartement des images, presque double de celui de la fig. 13, que la vitesse a beaucoup augmenté. Quelques décharges isolées dans la masse gazeuse se voient également, progressant aussi dans le sens anode-cathode.

Cette fragmentation devient très franche quand on augmente beaucoup la fréquence, et paraît tenir à la modification subie par le gaz.

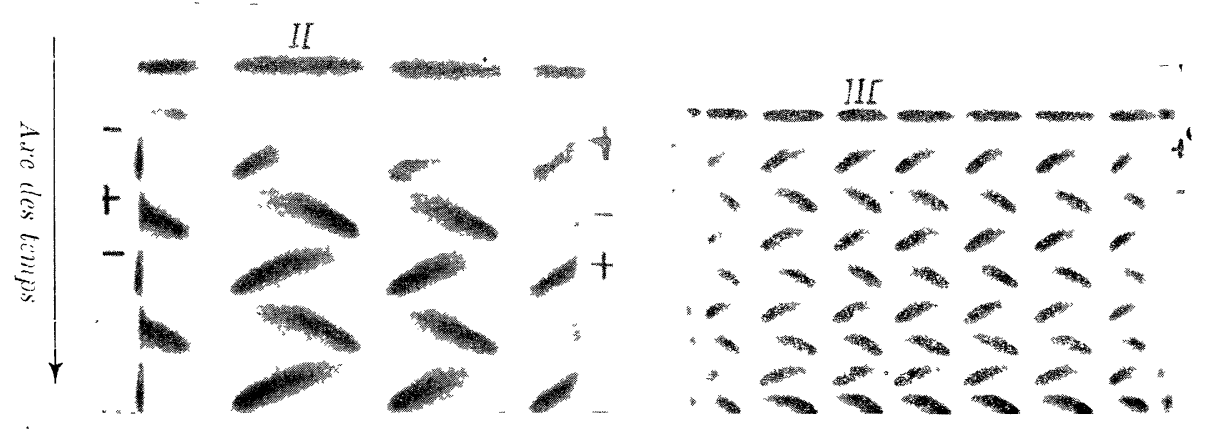

Fıg. 15. - Fragmentation de la lumière positive. -- Courant alternatif sans soupape : tubo de 5 centimètres: pression, 15 millimètres; à gauche, distance des électrodes : 47 centimètres; à droite, distance des électrodes : 90 centimètres.

Tous les segments s'allument simultanément et dans chacun d'eux la lumière progresse dans le sens anode-cathode, sens ici alternatif.

La fréquence (42 périodes) est la même que pour la fig. 14. On voit que la vitesse de progression de la décharge est ici beaucoup moindre.

On l'obtient aussi très facilement, avec le courant à 42 périodes, en laissant passer les deux alternances, de manière à réduire beaucoup le temps écoulé entre deux décharges. On obtient ainsi le résultat de la $f g .15$ sur laquelle on voit, en haut, l'image sur plaque immobile, c'est-à-dire l'aspect du tube, et au-dessous la série chronographique montrant nettement que les vitesses sont très faibles et dirigées dans 
le sens anode-cathode, sens ici alternatif. La fréquence étant doublée, l'ionisation est plus intense, les têtes des diverses colonnes positives ont seules assez d'éclat pour impressionner la plaque, et les images sont des lignes, ou plutôt des bandes inclinées.

Quand on fait cette expérience en commençant pár un courant intense, la colonne positive est continue d'aspect. Si on diminue l'intensité, des nodosités apparaissent comme sur un fil de platine chauffé presque à la température de fusion, puis la fragmentation se produit, donnant l'illusion d'une veine liquide qui se rompt quand on cherche à l'amincir.

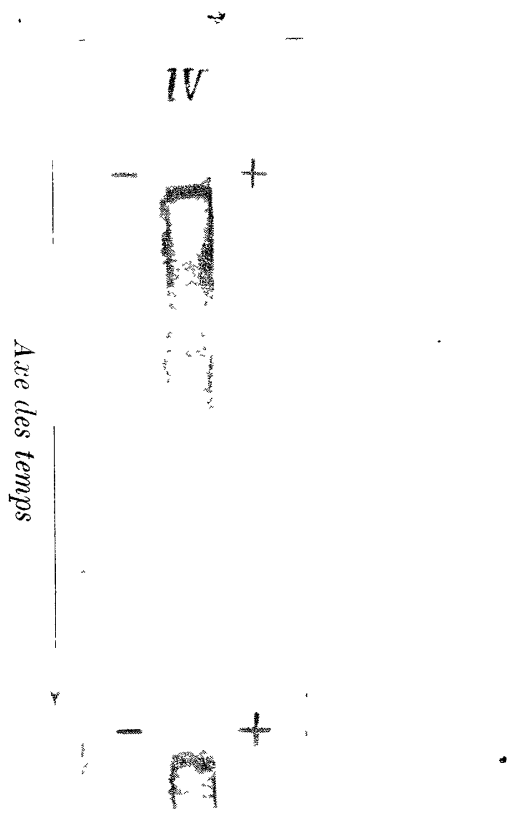

Fig. 16. - Arc à haut voltage à la pression atmosphérique. - Courant alternatif avec soupape (fréquence 42). Entre les deux décharges de même sens on voit un trait pâle (aigrette anodique), indiquant ce que laisse passer la soupape dans le sens où elle réagit. Distance des électrodes 1 centimètre.

Ce n'est pas seulement dans les tubes de Geissler que s'observe l'établissement de la colonne anodique dans le sens anode-cathode; le phénomène est exactement le même à la pression ordinaire et la vitesse est de même ordre ( $f g$. 16).

Il est intéressant de photographier sur plaque mobile la décharge 
d'un condensateur ( $\frac{1}{2}$ à 1 microfarad $)$. On obtient alors les résultats reproduits par la fig. 17. Pour de faibles charges, la lumière positive s'allonge à partir de l'anode, conserve un instant une longueur constante (régime permanent), puis disparaît en laissant subsister cepen-

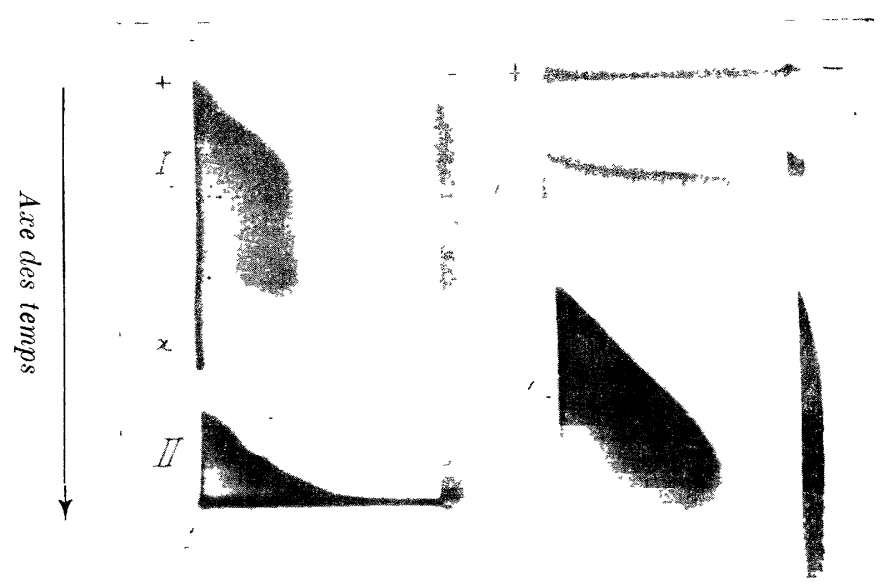

Fig. 17. - Chronophotographues ubtenues avec le dispositıf de la fig. 1 (décharge d'un condensateur). - Distance des électrodes, 10 centimètres ; pression, 15 millimètres.

I. 2400 volts. - Phénomène de Geissler avec régime permanent. Décharge visiblement incomplète du condensateur.

II. 2500 volts. - Phẻnomene de Geissler suivi d'un arc déchargeant complètement le condensateur et èteignant la lumière négative.

III. 3000 volts sans résistance. - Durée inappréciable.

IV. 3000 volts, 200 à $300 \mathrm{ohms.} \mathrm{-} \mathrm{Durée} \mathrm{notable.}$

V. 3000 volts, 5000 ohms. - Suppression de l'arc et retour au cas $n^{\circ} \mathrm{I}$.

dant à l'anode une aigrette se traduisant sur l'épreuve par un trait $a$, et on voit ainsi que le condensateur ne s'est pas entièrement déchargé.

Pour une charge plus forte ( $f g .17$, II), l'intensité arrive à la valeur qui permet l'allumage de l'arc, celui-ci donne un trait lumineux perpendiculaire à l'axe des temps, donc de durée inappréciable, et, après son passage, il ne subsiste pas d'aigrette aux électrodes; en outre, la gaine négative disparaît à l'instant où l'arc se produit.

Le numéro III de la même figure montre le trait de feu étabḷi plus rapidement encore (charge à 3000 volts pour 10 centimètres d'étincelle). La durée est inappréciable. Au numéro IV on voit une décharge obtenue avec le même voltage, mais en interca- 
lant une résistance : la durée devient mesurable et on voit que le trait de feu part de l'anode.

Enfin, au numéro $\mathrm{V}$, la résistance intercalée étant considérable (ॐ000 ohms), l'intensité n'a pu atteindre la valeur disruptive et on retrouve l'aspect du numéro I(1).

On voit ainsi qu'il n'existe aucune différence essentielle entre les décharges avec ou sans espace de Faraday. L'une et l'autre partent de l'anode, et de leur intensité seule dépend la possibilité d'átteindre la cathode.

\section{PhÉNomènes MÉcaniques. - expérience de Melde.}

La cohésion, apparente ou réelle, de la lumière positive et la tension qu'il faut lui attribuer pour expliquer la déviation magnétique permettent de prévoir que ce conducteur peut être traité, au point de vue mécanique, comme un fil matériel tendu.

La condition essentielle à réaliser est évidemment de donner à ce conducteur une existence permanente ; un courant continu serait sans doute parfait à ce point de vue, mais l'expérience a prouvé qu'un courant intermittent peut suffire, pourvu que les intermittences se succèdent avec une fréquence très élevée ; la chaîne anodique n'a pas le temps de se disloquer dans l'intervalle de deux décharges.

Ce résultat a été obtenu en employant le courant d'un alternateur à 500 périodes que M. H. Abraham a bien voulu mettre à ma disposition, ce dont je suis heureux de le remercier. Avec un transformateur et une soupape on obtient ainsi des décharges séparées par des intervalles peu supérieurs à $\frac{1}{1000}$ de seconde, ce qui est presque la continuité.

Cette fréquence élevée permet d'atteindre facilement de hauts voltages avec une simple bobine de Ruhmkorff comme transformateur, et l'emploi de pressions élevées ( 2 à 8 centimètres de mercure) devient possible. Dans ces conditions, l'effet d'écran produit par les

(1) Dans mes premières expériences, j'avais attribué ce ralentissement à la selfinduction. J'ai vérifié qu'une résistance purement ohmique produit les mèmes effets. Mais il est évident que les résultats seraient les mêmes avec un circuit peu résistant à grande constante de temps, la diminution d'intensité suffisant à empêcher l'établissement de l'arc. 
parois du tube à gaz raréfié devient insignifiant; tout se passe comme si on opérait à l'air libre.

On observe ainsi très aisément l'attraction de la chaîne anodique par un objet chargé négativement et placé à l'extérieur du tube. Il suffit même d'approcher la main pour attirer cette chaîne, comme la feuille d'or d'un électroscope dépourvu de cage protectrice, et on la fait aisément osciller à la manière d'une corde librement suspendue, l'anode étant placée à la partie supérieure du tube disposé verticalement.

On est ainsi conduit à réaliser l'expérience de Melde en prenant comme corde vibrante la lumière positive. Il suffit pour cela d'agiter l'extrémité cathodique en faisant osciller soit la cathode, montée par exemple sur une lame de ressort (1), soit une fourche métallique extérieure portée par une lame flexible et dont les branches attirent la lumière positive alternativement à droite et à gauche, la fourche étant reliée au sol ou à la cathode de la source.

En donnant à l'extrémité cathodique de la chaîne anodique une impulsion brusque aller et retour, on obtient une onde transversale qui se propage jusqu'àl'anode, simulant exactement ce qu'on observe dans l'expérience classique de la corde suspendue.

$\mathrm{Si}$ on fait osciller régulièrement cette même extrémité cathodique, on a les aspects instantanés représentés par la fig. $18\left(\mathrm{n}^{\circ} \mathrm{I}\right)$.

Pour une période convenable, on obtient des nœuds et des ventres (fig. 18, $\left.\mathrm{n}^{\circ} \mathrm{II}\right)$, comme dans une expérience de Melde qui, toutefois, serait mal réglée.

Le numéro III de la même figure montre le résultat obtenu à la pression de ă centimètres, l'agitation étant faite par la fourcheoscillante dont la période est aisée à régler. La photographie a été faite avec une pose simplement courte, chaque épreuve donnant ainsi toutes les positions comprises entre deux phases distinctes.

Le numéro IV est la photographie obtenue dans les mêmes conditions, mais sur plaque mobile. On voit toutes les positions successives de la chaîne oscillante. Il suffirait de calquer les diverses

(1) Pour les vibrations rapides, la cathode était une tige d'aluminium montée à l'extrémité d'une lame d'acier encastrée, et chargée d'une masse assurant l'entretien du mouvement pendant une dizaine de secondes. L'impulsion était donnée par un choc sur le tube ou avec un petit aimant. Les oscillations lentes étaient produites par une cathode montée entre pivots à peu près comme une pendule de Kater. La partie inférieure était lestée par une masse de fer permettant la manœuvre à l'aide d'un aimant. 
images et de les placer dans un kinétoscope ou cinématographe pour

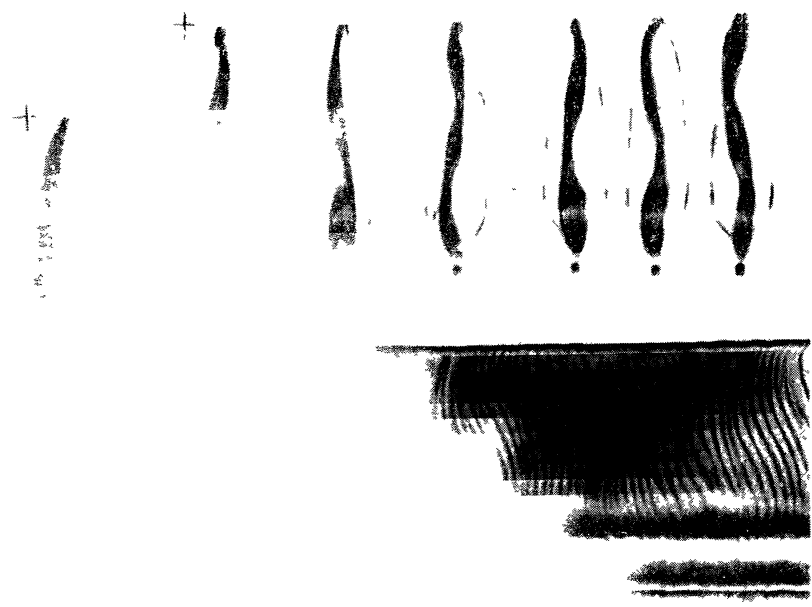

FIG. 18.

reconstituer le mouvement, qui est tout à fait semblable à celui d'une corde vibrante.

Particularités de l'allumage à haute fréquence.

L'épreuve $\mathrm{n}^{\circ}$ IV de la $f i g .18$ a été obtenue avec une pose totale voisine d'une seconde. Elle devrait contenir environ 500 images de la chaîne anodique. Or on en compte moins de cent. On a l'explication de cette singularité en répétant l'expérience avec un déplacement plus rapide de la plaque (fig. 19). On voit alors que la chaîne oscillante ne s'illumine pas en entier à chaque période, et qu'il faut 12 décharges pour avoir une image complète de cette chaîne. Il est tout à fait manifeste sur la $f g .19$ que l'allumage est, à l'in- 
PASSAGE DE L'ÉLECTRICITÉ DANS LES GAZ 35 כ termittence près, tout à fait semblable à celui d'une traînée de poudre : à un instant donné, il n'y a de lumière que sur une très petite

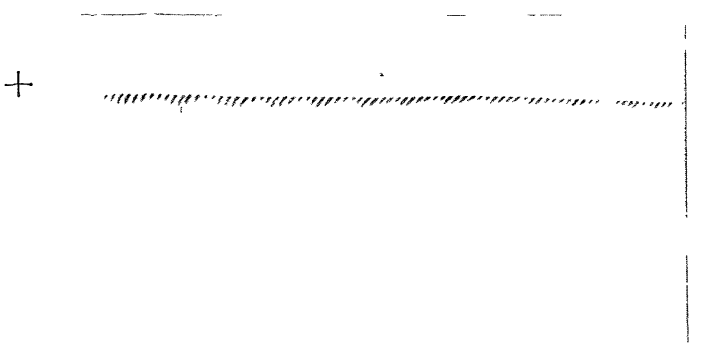

FIG. 19.

longueur, ce qui est brûlé n'en émettant plus et ce qui est intact n'en donnant pas encore.

Le phénomène est donc le suivant : la chaîne anodique est constituée, déviable à l'aimant ou par attraction électrostatique, mais invisible encore ; puis l'émission de lumière commence à l'anode et s'étend progressivementle long de la chaîne, l'éclat étant maximum sur le front d'attaque, très faible sur la partie déjà parcourue et en quelque sorte brûlée. La vitesse de progression étant très petite, le trajet parcouru pendant la durée d'une décharge (moins de $\frac{1}{\mathbf{1 0 0 0}}$ de seconde) n'est que de 20 millimètres environ. A la décharge suivante, le gaz brûlé n'est pas revenu à l'état initial, et tout se passe comme s'il n'y avait pas eu d'interruption; l'incandescence repart du point où elle avait cessé, parcourt un nouveau trajet de 20 millimètres, qui chevauche un peu sur le précédent, s'éteint encore une fois, et ainsi de suite jusqu'à l'espace de Faraday. Le trajet total est donc parcouru en plusieurs étapes au lieu de l'être d'un seul coup; ceci était à prévoir, la fréquence du courant ne pouvant évidemment augmenter la vitesse de progression de l'incandescence du gaz.

Tout cela est aisé à vérifier en photographiant, sur plaque mobile, 
le début de l'allumage, la chaîne anodique étant laissée en repos $\left(f i g .18, \mathrm{n}^{\circ} \mathrm{V}\right.$, et fig. 20 ). On voit alors très nettement que cet allu-

FIG. 20 .

mage se fait par bonds successifs, et qu'il a fallu (dans cette expérience) sept périodes pour atteindre l'espace de Faraday.

Mais, pendant ce temps relativement grand $\left(\frac{7}{500}\right.$ de seconde $)$, le gaz modifié a pu se reconstituer, etl'allumage recommence à l'anode pour donner une seconde fois la même série d'échelons.

On voit, en outre, qu'au bout d'un grand nombre de périodes la luminescence devient extrèmement faible. Cet affaiblissement n'avait pas lieu quand la chaîne anodique oscillait; il faut donc l'attribuer à l'accumulation du gaz modifié sur le trajet fixe de la décharge. Ici encore on voit que l'ionisation n'est pas favorable à l'émission de la lumière.

\section{INFLUENCE DE LA DISTANCE DES ÉLECTRODES.}

Dans un large tube AC ( $f g . \mathbf{2 1}$ ), disposons deux électrodes planes de même diamètre que le tube, et dont l'une sera mobile à coulisse (1), l'anode par exemple. Supposons la distance des plateaux

(1) L'extrémité libre de la tige $t$ est terminée par un petit cylindre de fer. Le déplacement se fait au moyen d'une courte bobine qu'on fait glisser sur le tube de verre contenant la coulisse. Il est avantageux que la bobine soit parcourue par un courant alternatif, les vibrations produites par ce courant supprimant les frottements au départ.

La cathode doit être absolument jointive et, pour cela, munie en arrière d'un large rebord; sans cette précaution, la décharge peut en faire le tour. 
d'abord très grande, et le vide tel que l'épaisseur $a b$ de l'espace obscur de Hittorf soit petite comparée au diamètre du tube (5 à 6 millimètres pour un diamètre de 6 centimètres au moins).

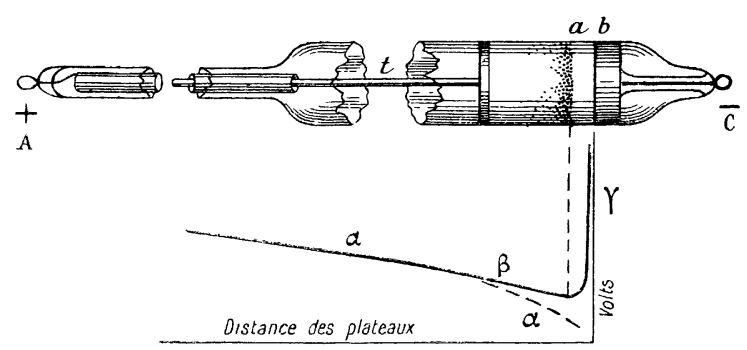

FIt. 21 .

Faisons passer dans l'appareil un courant continu et rapprochons l'anode de la cathode en réglant le voltage de manière à maintenir l'intensité constante.

Le mouvement de l'anode raccourcit d'abord la colonne positive par son extrémité anodique sans modifier les autres parties de la décharge. Comme le gradient est constant dans la lumière positive, la décroissance du voltage aux électrodes se fait linéairement, et il en est ainsi jusqu'à l'espace de Faraday. A partir de ce moment on est dans la région où, l'ionisation cathodique supprimant la lumière positive, le courant passe par convection seulement. Le rapprochement de l'anode a pour effet de raccourcir le chemin que les ions doivent parcourir. et, pourvu que le champ demeure constant, le courant ne variera pas. Le voltage diminuera encore linéairement, mais suivant une loi différente de la précédente.

Cette décroissance continuera jusqu'à l'espace obscur de Hittorff, mais un autre phénomène interviendra alors : j'ai montré que tout obstacle placé dans cet espace gêne l'alimentation de la cathode par l'afflux cathodique $\left({ }^{1}\right)$. Le débit, proportionnel au nombre des particules constituant l'afflux, diminuera donc et, pour le maintenir constant, il faudra relever le voltage aux électrodes; on obtiendra ainsi

(1) En particulier, si le tube est obturé par un diaphragme perforé placé dans l'espace obscur, l'émission cathodique se fait presque uniquement par les points situés en face des centres des ouvertures du diaphragme.

J. de Phys., 4e série, t. VII. (Mai 1908.) 
la courbe représentée au-dessous du tube sur la même figure ( $\left.{ }^{1}\right)$.

11 résulte de ce qui précède que la différence de potentiel nécessaire au passage de la décharge entre deux plateaux est minima quand la distance des plateaux est exactement égale à l'épaisseur de l'espace obscur de Hittorff.

On verra plus loin que la décharge ne peut avoir lieu qu'à partir d'une intensité minima. D'autre part, l'épaisseur de l'espace obscur augmente quand l'intensité diminue. On est ainsi conduit à l'énoncé suivant :

Le voltage nécessaire à l'établissement d'une décharge électrique entre plateaux parallèles est minimum quand la distance des plateaux est égale à l'épaisseur d'espuce obscur qui correspond au courant minimum exigé par la décharge $\left(^{2}\right)$.

Construite pour ce courant critique, la courbe précédente ne peut être qu'identique à la courbe dite de Paschen.

D'après ce qu'on vient de voir, cette courbe résume les lois de trois phénomènes essentiellement distincts : la lumière positive, la convection dans l'espace de Faraday, l'alimentation de la cathode (régions $\alpha, \beta, \gamma$ de la courbe); on comprend qu'il soit difficile de traduire cette courbe par une fonction simple.

Il est d'ailleurs facile de vérifier que le relèvement du potentiel explosif le long de la branche $\gamma$ est bien dû aux phénomènes cathodiques. Il suffit de prendre pour électrodes deux plateaux minces ( 1 millimètre) d'un diamètre très légèrement inférieur à celui du tube. Quand les plateaux sont à une distance inférieure à l'épaisseur de l'espace obscur, la décharge allonge d'elle-même son trajet en contournant la cathode, et jamais en contournant l'anode.

Dans l'expérience précédente, on pourrait maintenir le voltage constant et obtenir l'extinction de la décharge en rapprochant les

(1) Il est commode de réaliser un courant constant au moyen d'une source à très haut voltage, en série avec une très forte résistance. Dans ces conditions, le tracé de la courbe est très facile; il suffit de lire à tout instant les indications d'un voltmètre statique relié aux points A et $\mathrm{C}$.

() 11 conviendrait de dire : «Entre plateaux parallèles indéfinis. » Pratiquement l'épaisseur $a b$ de l'espace obscur doit être négligeable vis-à-vis du diamètre des plateaux. Si elle est trop grande, le minimum du voltage a lieu pour une distance des plateaux supérieure à $a b$. Cela résulte de l'électrisation positive perturbatrice des parois, électrisation qui resserre, comme je l'ai démontré, l'afflux cathodique, diminue la section utile de la cathode et réduit le débit. Tout se passe comme si une anode annulaire était placée dans l'espace obscur. Il faut donc rendre insignifiante cette action des parois, et cette condition serait précisément réalisée avec des plateaux de largeur infinie. 
électrodes, ou l'allumage en les écartant (branche $\gamma$ ). Cette réversibilité ou réciprocité caractérise le phénomène de Geissler. Supposons maintenant qu'on laisse l'intensité prendre une valeur telle que l'arc s'établisse. Il n'y aura plus alors d'espace de Faraday ni de phénomènes cathodiques, et la branche $\gamma$ de la courbe disparaitra ainsi que la partie $\beta$. On pourra encore observer l'allumage par écartement des électrodes, parce que, dans l'établissement d'une décharge quelconque, l'intensité part nécessairement de zéro et, qu'avant d'arriver à l'arc, on passera par le phénomène de Geissler. Mais, une fois l'arc établi, on ne pourra pas l'éteindre en rapprochant les électrodes, et la réversibilité dont il était question plus haut n'existera plus. La courbe se réduira à la branche $\alpha$, qui se prolongera jusque vers l'origine.

Ainsi la courbe qui représente la relation entre le potentiel explosif et la distance des électrodes est, aux retards près, applicable aussi bien à l'extinction qu'à l'établissement du phénomène de Geissler. Elle ne s'applique pas à l'arc une fois établi et se réduit à la branche qui correspond à la lumière positive.

\section{ROLE PRÉPARATOIRE DES IONS.}

Il est tout à fait manifeste qu'une ionisation intense gêne l'étincelle électrique, supprime par exemple la lumière positive, empêche l'établissement de l'arc et transforme la décharge lumineuse en un courant de convection obscur. Il n'est cependant pas niable qu'une légère ionisation abaisse le potentiel explosif et paraît même nécessaire à l'établissement de la décharge.

D'autre part, c'est généralement par défaut d'intensité, et non insuffisance de voltage, qu'une décharge électrique s'éteint('). C'est précisément cette condition d'un minimum d'intensité qui fait disparaître la contradiction.

Reprenons le tube de la fig. 21 et relions ses électrodes à une batterie d'accumulateurs par l'intermédiaire d'une résistance très forte (supérieure à 10 mégohms) et d'un galvanomètre.

L'électrode mobile étant, par exemple, d'abord trop près de la cathode, éloignons-la progressivement de celle-ci : à un certain

(1) On a vu en effet que, sauf pour les tubes de Crookes où la lumière positive manque ou n'est pas prépondérante, le voltage baisse à mesure que l'intensité augmente. 
moment le galvanomètre accuse le passage d'un très faible courant qui augmente si on fait croître la distance des plateaux et diminue dans le cas contraire. L'intensité peut ainsi varier de quelques centmillionièmes à quelques millionièmes d'ampère et suit exactement, d'une manière en quelque sorte réversible, les variations de distance des électrodes, sous la seule condition qu'on ne dépasse pas une certaine intensité.

- Si on place le tube dans un champ magnétiquelongitudinal, variable à volonté, le courant augmente ou diminue en suivant exactement les variations de la force magnétique. Un champ transversal affaiblit le courant ou même le supprime.

- Il est tout à fait évident qu'on est en présence d'un courant de convection. Des ions sont produits, transportent l'électricité d'une électrode à l'autre, mais sont en partie arrêtés par les parois du tube. Le champ longitudinal oblige ces ions à décrire des hélices et les canalise ainsi dans des tubes de force. La déperdition latérale diminue et le courant augmente. Comme on doit s'y attendre, cette augmentation cesse quand tous les ions sont captés, c'est-à-dire à partir d'une certaine valeur, évidemment faible, du champ magnétique; quelques dizaines d'unités suffisent pour cela. Un champ transversal rejette au contraire les ions sur les parois et supprime le courant. Ces propriétés sont absolument caractéristiques de cette phase de la décharge.

Supposons maintenant qu'en écartant les électrodes, ou au moyen du champ magnétique, on dépasse une certaine intensité, aussitôt le courant se met à augmenter de lui-même plus ou moins vite; sa valeur devient dix ou vingt fois supérieure à la limite qu'on venait de franchir, et en même temps le voltage aux électrodes baisse énormément par suite de la chute de tension dans la résistance; sans cette dernière, on arriverait généralement à l'arc.

A partir de ce moment, les propriétés du tube en activité sont complètement transformées, un champ longitudinal n'a plus qu'une action insignifiante sur l'intensité : de même pour le champ transversal. Le phénomène est donc tout autre que précédemment, et par conséquent on n'a plus affaire à un pur courant de convection, on est en présence du phénomène de Geissler, d'ailleurs visible dans l'obscurité.

Remarquons maintenant que cette transformation, due à un accroissement d'intensité, a été accompagnée d'une forte baisse de voltage 
PASSAGE DE L'ÉLECTRICITÉ DANS LES GAZ 361 (50 0/0 par exemple). Ce voltage était donc, dans la première partie de l'expérience, plus que suffisant pour que la décharge soit possible. L'ionisation existait, puisque le courant passait; toutes les conditions requises semblaient donc réalisées; cependant la décharge ne s'établissait pas. Il paraît donc évident qu'une certaine intensité est nécessaire pour que la décharge se constitue, et qu'il y a en particulier un véritable seuil pour la formation de la chaìne anodique. On a déjà vu quelque chose d'analogue à propos des phénomènes précurseurs.

Cette conclusion paraît d'autant plus naturelle que la résistance de la chaine anodique possède la remarquable propriété de diminuer à mesure que le courant augmente, jusqu'à devenir presque nulle quand l'arc est établi.

Ainsi l'ionisation est bien nécessaire pour préparer la route de l'étincelle, le courant de convection qu'elle produit servant à franchir le seuil d'établissement de la décharge proprement dite.

En résumé, la lumière positive ou anodique n'est pas un ensemble de particules indépendantes les unes des autres; c'est un objet dont toutes les parties sont liées entre elles et qui se comporte comme un conducteur flexible et extensible.

Cet objet, comparable peut-être à un tourbillon, ou à une chaîne de limaille dans un champ magnétique, ou à une veine fluide, est d'autant plus important, plus stable et plus conducteur que le courant est plus intense; il ne se constitue qu'à partir d'une certaine intensité, d'où la nécessité d'un amorçage par le courant de convection résultant d'une ionisation préalable. Enfin il est détruit ou désagrégé par une ionisation excessive.

L'arc électrique, l'étincelle oscillante d'un condensateur, la luminescence instantanée d'une ampoule sans électrodes dans un champ croissant sont des décharges intenses réduites par cela même à la lumière positive sans gaine négative ni chute cathodique.

Dans un tube de Crookes, tout se réduit au contraire à des phénomènes cathodiques.

Le phénomène de Geissler est une décharge assez peu intense pour que l'ionisation cathodique puisse désagréger la lumière positive près de la cathode (espace obscur de Faraday). C'est la décharge au maximum de différentiation.

Le transport de l'électricité par simple convection, c'est-à-dire par des ions libres, ne produit pas de lumière. 